\title{
IMPACTO DE INTERVENCIÓN TEMPRANA A CUIDADORES DE PACIENTES SECUELADOS DE UN ACCIDENTE CEREBRO VASCULAR. ESTUDIO PILOTO.
}

\section{IMPACT OF EARLY INTERVENTION FOR STROKE CAREGIVERS. A PILOT STUDY.}

Evelyn Álvarez TO ${ }^{1,2,3}$, Sebastián Gallegos TO, Eduardo Romero TO, Carolina Moraga TO, Marcela López TO, Consuelo Estadella TO, Valentina Viviani TO, Denisse Ortega TO, Daniela Venegas TO, Ingrid Pérez EU ${ }^{l}$, Katherine Vargas $M D$, Sergio Illanes $M D^{I}$.

\section{RESUMEN}

Introducción: Los cuidadores de personas secueladas de un Accidente Cerebro Vascular $(\mathrm{ACV})$, perciben que durante los primeros meses carecen de la información necesaria sobre el proceso de su familiar, afectando sobre su estado afectivo y percepción de estrés.

Objetivo: Evaluar la intervención realizada a cuidadores de pacientes secuelados de un ACV como factor protector en el estado afectivo y de carga en los cuidadores.

Sujetos y métodos: Se reclutaron cuidadores directos, de personas con secuelas de ACV, según Escala de Rankin durante el período hospitalario. Se realizo un taller teóricopráctico, consistentes en 4 sesiones; 3 durante el periodo hospitalario del usuario y 1 dentro del primer mes posterior al alta, los temas tratados eran organización y distribución de la rutina, integración de rutinas de tiempo libre y relajación y manejo del paciente en el hogar. Se evalúo a los cuidadores con escalas diagnósticas de carga emocional (Zarit, Indice de Esfuerzo del Cuidador) y ansiedad (Goldberg).

Resultados: Luego del taller hubo una reducción significativa de $20 \%$ en el número de cuidadores con depresión $(\mathrm{p}=0,036)$ y $37 \%$ en el número de cuidadores con ansiedad $(\mathrm{p}=0,034$.). Asimismo hubo una disminución significativa en el puntaje de sobrecarga emocional del cuidador medido mediante la escala de Zarit $(p=0,044)$.

Conclusiones: La intervención evaluada en el presente estudio piloto parece ser una medida que contribuye en disminuir la sobrecarga emocional y ansiedad del cuidador.

Palabras claves: Accidente Vascular Encefálico, Autocuidado, Carga, Cuidadores, Educación.

1. UTAC, Departamento de Neurología/Neurocirugía Hospital Clínico Universidad de Chile

2. Escuela de Terapia Ocupacional, Facultad de Medicina Universidad de Chile

3. Departamento de Fisiatría, Hospital Clínico Universidad de Chile 


\section{ABSTRACT}

Background: Caregivers of ischemic stoke damaged patients feels during the first months after the event a lack of information on how to help their relative, affecting the sense of stress and anxiety. We made an intervention to caregivers of the stroke unit of the University of Chile Hospital, in 2008.

Objective: Determine whether the intervention to caregivers of stroke damaged patients is a protective factor in their emotional state and stress on caregivers.

Subjects and methods: Direct healthy caregivers were recruited with a severe impaired family member within the hospitalization period. Intervention was conducted in 4 sessions, 3 during the hospital period and 1 in his home within the first month after discharge, where the issues discussed with caregiver was self-care and patient management. To measure the intervention we evaluated before the workshop and 2 months after the last session with some standardized tests (Zarit, Caregiver Stress Index) and anxiety (Goldberg).

Results: After the intervention there was a significant $20 \%$ reduction in the number of caregivers with depression $(\mathrm{p}=0.036)$ and $37 \%$ in the number of caregivers with anxiety $(\mathrm{p}=0.034$.). There was also a significant decrease in the score of caregiver's emotional overload measured by the Zarit scale ( $\mathrm{p}=0.044)$.

Conclusions: The intervention evaluated in this pilot study appears to be an effective measure in reducing the emotional overload and anxiety of the caregiver, with an evident improvement in their mood.

Key words: Stroke, Selfcare, Burden, Caregivers, Education 


\section{INTRODUCCIÓN}

Los Accidentes Cerebro Vasculares (ACV) representan la segunda causa de muerte a nivel mundial, alcanzando aproximadamente un tercio de mortalidad durante el primer episodio ${ }^{1}$. En Chile estos son la segunda causa de mortalidad y la primera de hospitalización en mayores de 65 años $^{2}$. Un alto porcentaje de los pacientes que sobreviven presentan alguna discapacidad, estimandose que entre un 25 y un $74 \%$ de ellos requieren algún tipo de ayuda para la realización de las actividades de la vida diaria ${ }^{3}$ (AVD). En la población chilena, un 63\% de los pacientes necesita algún tipo de ayuda a los 6 meses de evolución ${ }^{4}$ y a los 3 años un 49,1\% presenta algún grado de discapacidad ${ }^{5}$ generando un importante impacto económico y social. Esta dependencia suele implicar que algún miembro de la familia asuma el rol de cuidador informal, invertiendo la mayor parte de su tiempo en la asistencia de las AVD del paciente ${ }^{3.6}$.

Los cuidadores de personas con ACV perciben que la información entregada sobre la enfermedad, precauciones y cuidados requeridos es inadecuada e insuficiente, lo que genera una insatisfacción en el apoyo prestado por el equipo profesional ${ }^{7-10}$, esto conlleva desde concepciones erróneas hasta trastornos ansiosos y alteraciones del ánimo en los cuidadores, repercutiendo directamente sobre su calidad de vida, salud mental y sensación de carga ${ }^{11}$. Además se ha encontrado que la relación cuidador- paciente tiene un importante papel en el proceso de rehabilitación y en el bienestar de los sobrevivientes de un $\mathrm{ACV}^{8,9,12}$, es por eso que se ha relacionado que los cuidadores que presentan un estado depresivo y elevada sensación de carga pueden empeorar o desencadenar síntomas depresivos en su familiar y disminuir la adherencia y efectividad en proceso de rehabilitación ${ }^{13,14}$.

Debido a esa problemática, existe la necesidad de generar estrategias eficaces que minimicen la sensación de estrés, la carga y la ansiedad del cuidador ${ }^{15}$, especialmente durante los 3 primeros meses post-ACV que es cuando se presentan los mayores niveles de alteraciones en la salud mental en cuidadores que tienen en su cuidado a personas altamente dependientes ${ }^{12,16}$. 
El objetivo de nuestro trabajo es evaluar la eficacia de una intervención teórico-práctico impartida precozmente a cuidadores-familiares que se harán cargo de pacientes secuelados de un ACV luego de su alta hospitalaria.

\section{MATERIALES Y MÉTODOS}

El estudio es una serie de casos intervencional prospectiva no aleatoria, donde se enrolaron cuidadores desde la Unidad de tratamiento del ataque cerebral (UTAC) del Hospital Clínico de la Universidad de Chile, durante el año 2008 mientras su familiar se encontraba hospitalizado en la unidad. Para el trabajo se consideraron criterios tanto para los cuidadores como los pacientes, para los cuidadores se requirió que fueran cuidadores directo, tener entre 18 y 70 años, no presentar alguna enfermedad discapacitante (definido en la escala de Rankin 0 a 1), dos enfermedades crónicas o enfermedades de salud mental (alcoholismo, drogadicción o antecedentes psiquiátricos). En cuanto a los pacientes debían haber sido independiente antes del ACV (escala de Rankin 0 a 1) y al momento del alta debe presentar una discapacidad importante (escala de Rankin entre 2 - 5). La selección de casos fue consecutiva durante los meses de Abril a Diciembre 2008.

Antes de ingresar al estudio a todos los cuidadores se les informo y explico acerca del estudio, utilizando como estructura el consentimiento informado, aprobado por el comité de ética del Hospital Clínico Universidad de Chile.

\section{Protocolo de intervención}

Los cuidadores participaron en un taller, el cual constaba de 4 sesiones de caracter teóricopráctico; al inicio de cada sesión se preguntaba que conocían acerca de los temas propuestos (tabla 1), luego se discutían, uniendo los conocimientos de los participantes y el de los tratantes, finalizando con actividades practicas durante la misma sesión, sobre los temas desarrollados, las primeros 3 encuentros fueron realizadas mientras el paciente estaba hospitalizado.

La última sesión correspondió a una visita domiciliaria realizada durante el primer mes luego del alta hospitalaria, donde se buscó reforzar los contenidos en el taller, además de 
conocer problemáticas del cuidado, manejo ambiental y organización de la rutina, presentados durante ese periodo. Cada sesión tuvo 75 minutos de duración apróximadamente. Además se les entregó material escrito de apoyo que reforzaba lo aprendido (Figura 1).

Los contenidos tratados en las sesiones tuvieron dos ejes, uno relacionado con el apoyo del cuidado del paciente y el otro en el autocuidado del cuidador, donde se abordaron los siguientes temas: Definición de ACV y rehabilitación, precauciones en las alteraciones sensitivas y perceptuales en el hogar, técnicas básicas de manejo en las AVD (Higiene, alimentación, vestuario, transferencias), técnicas básicas de relajación, distribución y organización de la rutina y tiempo libre y por último riesgo ambiental y modificaciones ambientales.

\begin{tabular}{|c|c|}
\hline $\begin{array}{l}\text { Lugar donde se } \\
\text { realizo la sesión }\end{array}$ & Temas a tratar \\
\hline Hospital & $\begin{array}{l}\text { Definición de ACV y Rehabilitación. } \\
\text { Precauciones en las alteraciones sensoriales en el hogar. } \\
\text { Técnicas de relajación. }\end{array}$ \\
\hline Hospital & $\begin{array}{l}\text { Distribución de las tareas con la familia. } \\
\text { Técnicas de relajación y tiempo libre. } \\
\text { Transferencias seguras }\end{array}$ \\
\hline Hospital & $\begin{array}{l}\text { Técnicas de manejo en actividades de la vida diaria } \\
\text { (Vestuario, higiene, alimentación). } \\
\text { Técnicas de Relajación. }\end{array}$ \\
\hline Domicilio & $\begin{array}{l}\text { Riesgo ambiental y modificación ambiental (en visita } \\
\text { domiciliaria) }\end{array}$ \\
\hline
\end{tabular}

Tabla 1. Temas tratados en cada sesión.

Se describen a continuación los temas tratados en cada sesión. Se consideraron aspectos de la investigaciónacción participativa 

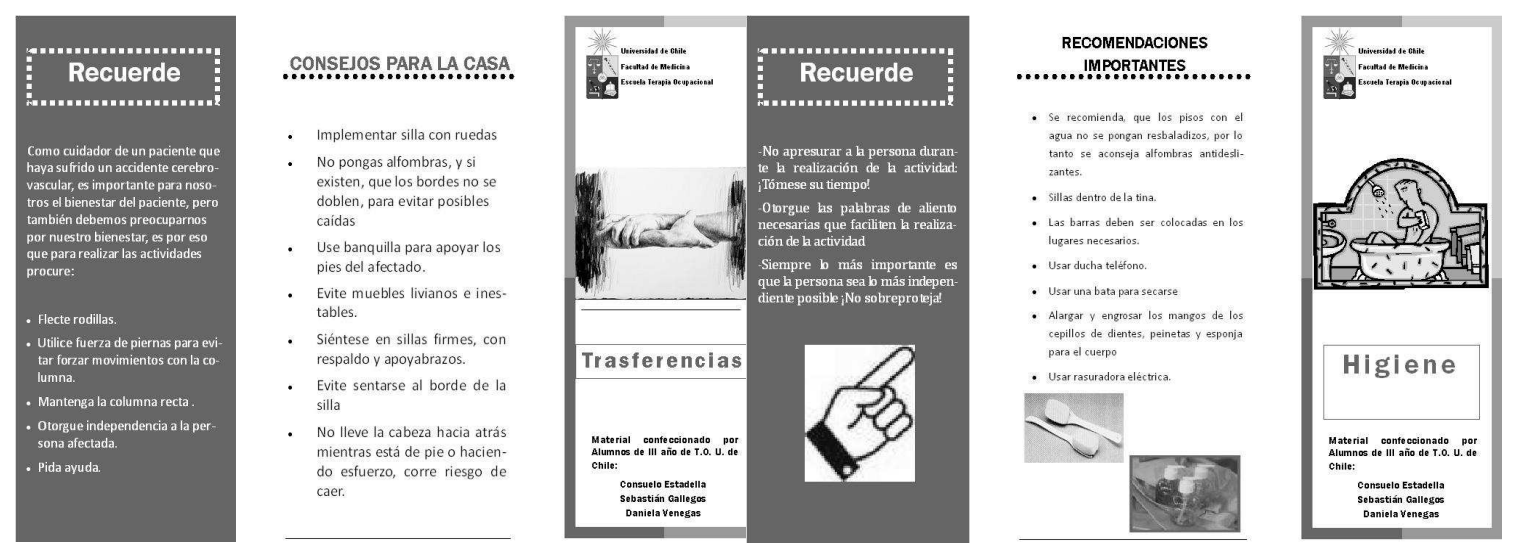

Figura 1. Material confeccionado.

Es un ejemplo material confeccionado para entregar en las sesiones

\section{Protocolo de evaluación}

A los cuidadores reclutados se les realizó una entrevista durante los primeros 15 días del ACV de su familiar y una segunda entrevista de seguimiento 2 meses después de haber participado en el taller de cuidadores. Las mediciones fueron realizadas a los pacientes y a los cuidadores, incluyendo además los datos demográficos. A cada paciente se la aplicaron las siguientes escalas de evaluación: a) Indice de Barthel (IB), mide el desempeño en 10 actividades básicas de la vida diaria, siendo divididos en dos grupos, actividades relacionadas con los cuidados personales (alimentación, aseo, baño, vestuario, manejo vesical e intestinal y uso del baño) y en actividades relacionadas con la movilidad (deambulación, traslados y uso de escaleras). El IB aporta información en la puntuación global y en las puntuaciones parciales para cada actividad, el rango global puede variar entre 0, completamente dependiente y 100 puntos, completamente independiente ${ }^{17}$ y b) Escala de Rankin modificada (ERm) mide la independencia y no la realización de tareas concretas $^{18}$, definiendo 6 niveles de discapacidad, comenzando desde 0 donde no existe ninguna discapacidad hasta 5 donde existe una grave discapacidad, que requieren cuidados de enfermería, siendo el nivel 6 para determinar la muerte ${ }^{19,20}$. 
A los cuidadores, se le realizaron las siguientes evaluaciones: a) Escala de sobrecarga del cuidador de Zarit (Caregiver Burden Interview) escala auto-administrada que mide la sobrecarga del cuidador, compuesta por 22 ítems, que miden el grado en que el cuidador percibe el impacto del cuidado (en las preguntas 1a 3,7,8,10 a 14,17,22), la carga o relación cuidador-paciente (las preguntas 4,5,6,9,18,19) y las expectativas en la capacidad de cuidar $(15,16,20,21)^{21}$. Utiliza 5 opciones que varían de 0 (nunca) a 4 puntos (siempre), los resultados se suman en un puntaje total, variando entre 0 y 88 , según el puntaje se determina el nivel de sobrecarga; escasa sobrecarga (0-20), Leve (21-40), Moderada (4160), Severa (61-88), b) Índice de esfuerzo del cuidador (IEC) (Caregiver Strain Index), cuestionario compuesto de 13 preguntas, con opción de respuestas dicotómicas (si /no), estas miden la carga relacionada al trabajo, financiamiento, tiempo, social y físico del cuidador. Se puede obtener un máximo de 13 puntos, desde 7 puntos se considera que el cuidador tiene un alto nivel de $\operatorname{carga}^{22,23}$ y c) Escala de Ansiedad y Depresión de Goldberg, consta de dos subescalas: una para la detección de la ansiedad (Goldberg A), y otra para la detección de la depresión (Goldberg D). Ambas escalas tienen 9 preguntas, pero las 5 últimas preguntas de cada escala sólo se formulan si hay respuestas positivas a las 4 primeras preguntas, que son obligatorias. En la Goldberg A, desde 4 o más se considera el punto de cohorte, en cambio en Goldberg D debe 2 o más, con puntuaciones tanto más altas más severo será el problema (siendo el máximo posible de 9 en cada una de las subescalas) ${ }^{24}$.

\section{Análisis estadístico}

Para el análisis estadístico se utilizó el programa SPSS v. 15.0. Debido al tamaño de la muestra, se usó la prueba $T$ student para una muestra, para determinar si existen diferencias significativas entre las medias en los dos momentos de evaluación. Todos se analizaron con un nivel de significación de 0,05. 


\section{RESULTADOS}

Un total de 17 duplas paciente/cuidador fueron reclutados para este estudio piloto, de los cuales 14 completaron el taller (tres sesiones hospitalarias, una visita domiciliaria) junto al protocolo de evaluación (Evaluación basal y a los 2 meses), ya que 2 pacientes fallecieron y 1 cuidador, no quiso continuar en el estudio. A continuación se muestran las características generales del grupo, tabla 2.

\begin{tabular}{|l|l|l|}
\hline & Pacientes & Cuidadoras/es \\
\hline Mujeres & $6(42 \%)$ & $12(85,7 \%)$ \\
\hline Edad, años (X+D.E) & $73,85 \pm 8,32$ & $54,42 \pm 12,82$ \\
\hline Años de escolaridad (X \pm D.E) & $9,14 \pm 4,5$ & $12,7 \pm 3,04$ \\
\hline & Relación cuidadoras/es con el paciente \\
\hline & Hija/o & $8(57.1 \%)$ \\
\hline & Esposa & $3(21 \%)$ \\
\hline & Nieta / sobrina & $2(14.2 \%)$ \\
\hline & Otro & $1(7.1 \%)$ \\
\hline
\end{tabular}

Tabla 2. Características Generales de la muestra

\section{Pacientes}

Las características generales de los pacientes del estudio se resumen en la tabla 3. En la evaluación basal $1 / 14$ pacientes $(0 \%)$ tuvieron una buena evaluación funcional (mRS < de 3 puntos), en cambio a los dos meses $5 / 14$ pacientes $(35,3 \%)$ mejoraron su rendimiento funcional global ( $p=0.0001$. Figura 2). Asimismo el puntaje de evaluación de AVD medido mediante BI al ingreso fue de 42,7 \pm 24 puntos, a los dos meses hubo una mejoría significativa llegando a los 72,7 $\pm 19,2$ puntos ( $T$ test, $p<0,01$. Figura 3).

\begin{tabular}{|l|l|l|r|r|r|}
\hline $\begin{array}{l}\text { ERm } \\
\text { inicial }\end{array}$ & $\begin{array}{l}\text { ERm } \\
\text { 2 meses }\end{array}$ & IB inicial & IB 2 meses \\
\hline & 5 & 4 & 0 & 20 \\
\hline 4 & 1 & 35 & 85 \\
\hline & 4 & 3 & 25 & 75 \\
\hline
\end{tabular}




\begin{tabular}{|r|r|r|r|r|}
\hline 3 & 3 & 85 & 90 \\
\hline 3 & 3 & 45 & 75 \\
\hline & 5 & 3 & 20 & 90 \\
\hline & 3 & 2 & 80 & 90 \\
\hline & 5 & 3 & 40 & 60 \\
\hline 4 & 3 & 15 & 50 \\
\hline & 4 & 3 & 30 & 55 \\
\hline & 2 & 2 & 50 & 80 \\
\hline & 4 & 1 & 85 & 95 \\
\hline & 4 & 2 & 30 & 90 \\
\hline & $\mathbf{X}+$ D.E) & 3 & 50 & 70 \\
\hline & $\mathbf{3 , 8 6} \pm \mathbf{0 . 8 7}$ & $\mathbf{2 . 5 7} \pm \mathbf{0 . 8 5}$ & $\mathbf{4 2 , 1 4} \pm$ & $\mathbf{7 3 , 2 1} \pm$ \\
& & & $\mathbf{2 6 , 1}$ & $\mathbf{2 0 . 9}$ \\
\hline
\end{tabular}

Tabla 3. Puntajes de Indice de BArthel (0-100)

y Escala de Rankin Modificada (0-6)

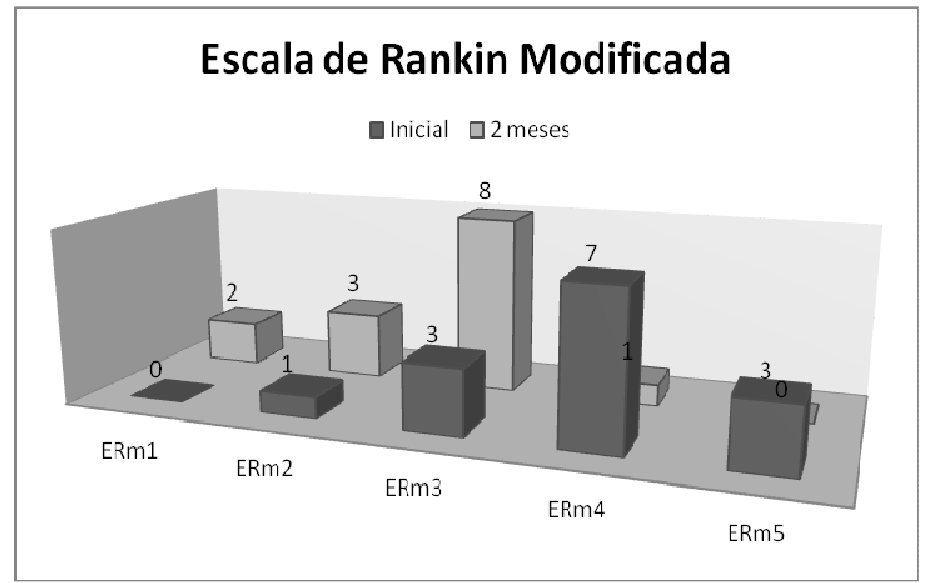

Figura 2. ERm. Gris oscuro evaluacion inicial, grus claro evaluacion post alta. Donde se observa que los pacientes se destribuyeron desde incapacidad severa (Erm 4) a moderada (Erm3)

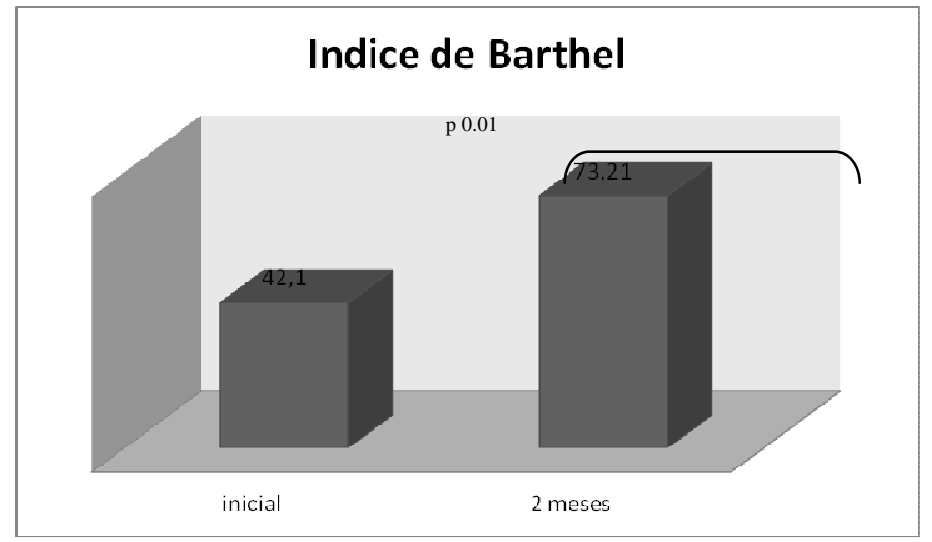

Figura 3. IB. Se observa que durante la primera evaluacion el grupo tuvo un promedio de 42,14 , mejorando su independencia a un total de 73,21 puntos

\section{Cuidadores}

En la evaluación basal 11/14 cuidadores $(78,5 \%)$ tuvieron un puntaje de Goldberg-D compatible con depresión (GD $\leq 2$ puntos). A los 2 meses 6/14 (42,8\%) pacientes tuvieron 
un buen desenlace en la escala de depresión ( $\mathrm{p}=0,016$. Figura 4). El puntaje de Goldberg-A de la evaluación basal fue compatible con ansiedad (GA > 3 puntos) en 6/14 cuidadores $(42,8 \%)$. A los 2 meses $2 / 14(14,28 \%)$ pacientes tuvieron un puntaje compatible con ansiedad $\left(X^{2} \mathrm{p}=0,065\right.$. Figura 5).

\begin{tabular}{|c|c|c|c|c|}
\hline & $\begin{array}{l}\text { GD } \\
\text { inicial }\end{array}$ & $\begin{array}{l}\text { GD } 2 \\
\text { meses }\end{array}$ & $\begin{array}{l}\text { GA } \\
\text { inicial }\end{array}$ & $\begin{array}{l}\text { GA } 2 \\
\text { meses }\end{array}$ \\
\hline & 1 & 1 & 0 & 4 \\
\hline & 4 & 7 & 8 & 6 \\
\hline & 6 & 2 & 0 & 2 \\
\hline & 4 & 1 & 3 & 0 \\
\hline & 5 & 3 & 0 & 1 \\
\hline & 1 & 2 & 5 & 1 \\
\hline & 3 & 1 & 4 & 2 \\
\hline & 3 & 0 & 5 & 0 \\
\hline & 7 & 2 & 1 & 1 \\
\hline & 4 & 1 & 3 & 2 \\
\hline & 2 & 4 & 7 & 1 \\
\hline & 5 & 1 & 5 & 0 \\
\hline & 1 & 4 & 1 & 1 \\
\hline & 6 & 2 & 1 & 1 \\
\hline X \pm D.E & $\begin{array}{r}3,71 \pm \\
1,97\end{array}$ & $\begin{array}{r}2.21_{ \pm} \\
1.81\end{array}$ & $\begin{array}{r}3,07 \pm \\
2,67\end{array}$ & $\begin{array}{r}1.57 \pm \\
1.6\end{array}$ \\
\hline
\end{tabular}

Tabla 4. Puntajes deEscala de Ansiedad (0-9) y Depresión de Goldberg (0-9).

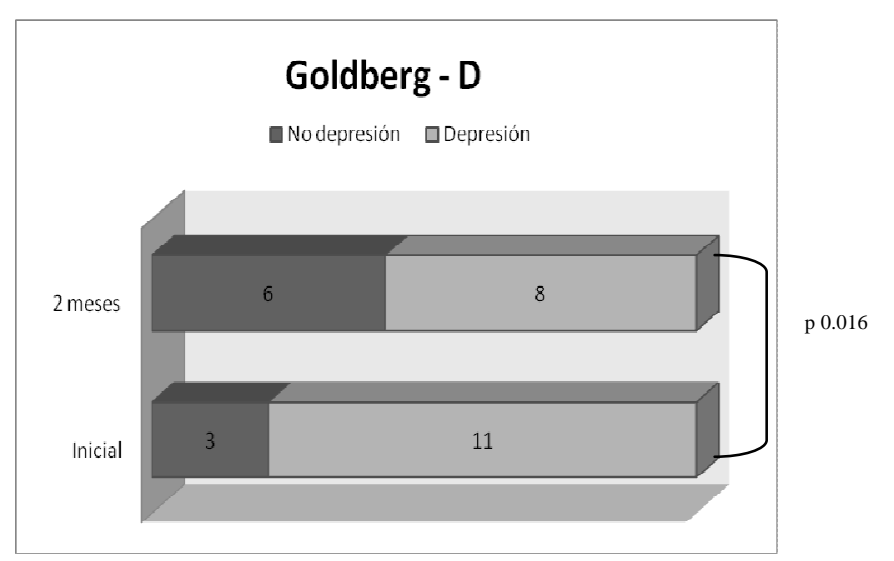

Figura 4. Depresión Goldberg. Gris oscuro sin depresión, gris claro con depresión.

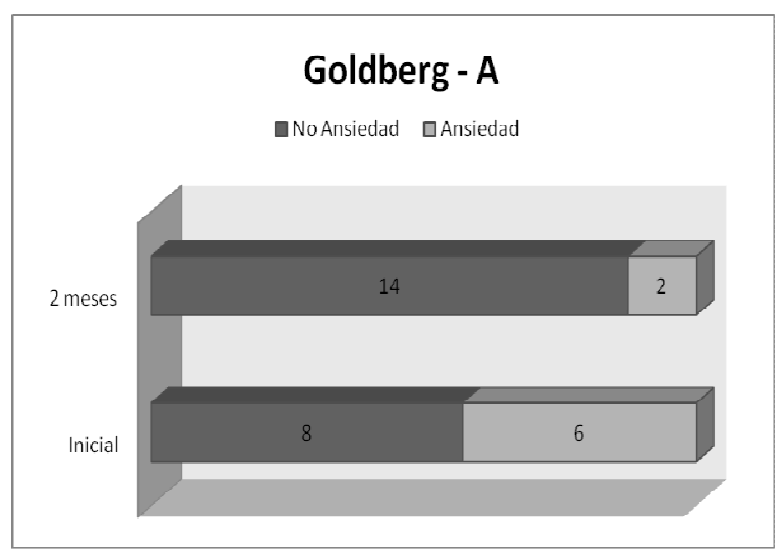

Figura 5. Ansiedad Goldberg. Gris oscuro sin ansiedad, gris claro con ansiedad. 
Asimismo el puntaje de evaluación de la sobrecarga emocional del cuidador medido mediante la escala de Zarit, tuvo un puntaje al ingreso de 33,4 $\pm 12,8$ puntos, a los dos meses hubo una mejoría significativa llegando a los 23,9 $\pm 13,5$ puntos ( $T$ test, $\mathrm{p}=0,0036$.

\section{Figura 6).}

\begin{tabular}{|c|c|c|c|c|}
\hline & $\begin{array}{r}\text { IEC } \\
\text { inicial }\end{array}$ & $\begin{array}{r}\text { IEC } 2 \\
\text { meses }\end{array}$ & $\begin{array}{c}\text { Zarit } \\
\text { inicial }\end{array}$ & $\begin{array}{l}\text { Zarit } 2 \\
\text { meses }\end{array}$ \\
\hline & 5 & 7 & 33 & 31 \\
\hline & 6 & 2 & 46 & 26 \\
\hline & 3 & 0 & 40 & 16 \\
\hline & 4 & 0 & 25 & 12 \\
\hline & 3 & 1 & 42 & 15 \\
\hline & 4 & 0 & 5 & 13 \\
\hline & 9 & 9 & 17 & 5 \\
\hline & 3 & 0 & 21 & 14 \\
\hline & 5 & 2 & 36 & 21 \\
\hline & 0 & 2 & 25 & 28 \\
\hline & 4 & 1 & 40 & 32 \\
\hline & 1 & 2 & 29 & 6 \\
\hline & 5 & 8 & 38 & 42 \\
\hline & 3 & 3 & 35 & 26 \\
\hline$X \pm D . E$ & $\begin{array}{l}3.9 \pm \\
2.16\end{array}$ & $\begin{array}{r}2,6 \pm \\
3.07\end{array}$ & $\begin{array}{r}30,86 \pm \\
11.26\end{array}$ & $\begin{array}{r}20,5 \pm \\
10.7\end{array}$ \\
\hline
\end{tabular}

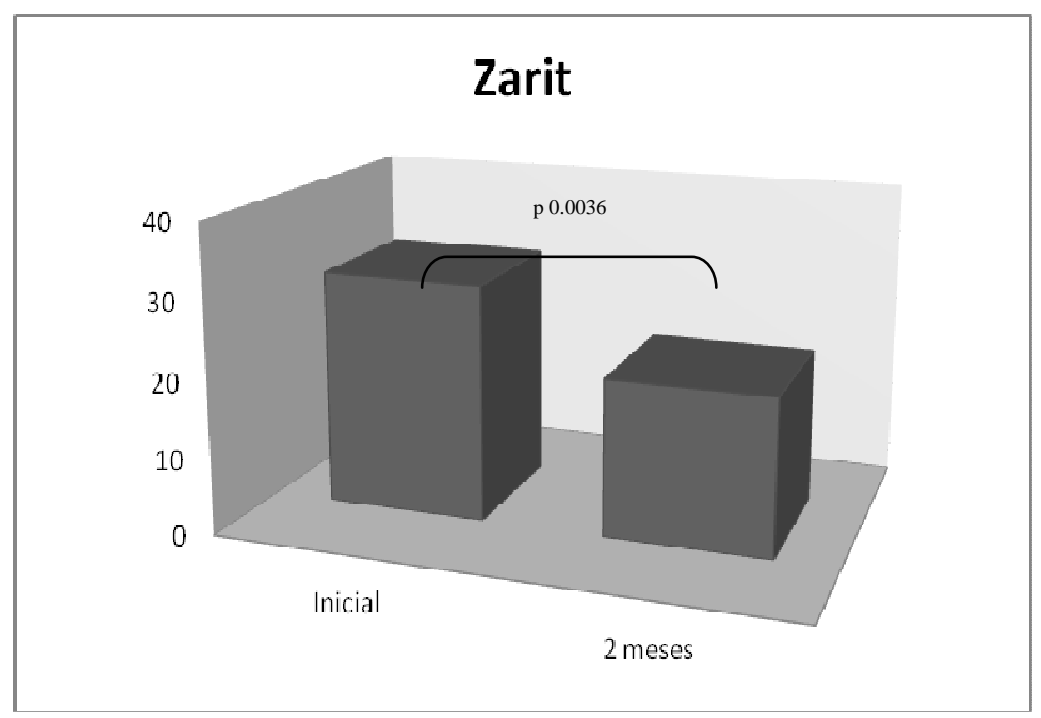

Figura 6. Escala de Sobrecarga del cuidador de Zarit (0-88).

Entre 0-20 escasa sobrecarga sobrecarga leve, 21-40 sobrecarga leve

Tabla 5. Puntaje evaluaciones de sobrecarga de cuidagor. 


\section{DISCUSIÓN}

Este trabajo está orientado a desarrollar una estrategia de apoyo al cuidador informal (generalmente un familiar) que debe asumir este rol, el cual muchas veces se debe enfrentar a este nuevo rol sin la preparación adecuada. La principal conclusión de nuestro trabajo es que la intervención parece ser una medida eficaz en disminuír la sobrecarga emocional ( $\mathrm{p}=$ 0,0036), junto con una evidente mejoría en su estado anímico ( $\mathrm{p}=0,016)$, lo que repercute en el pronóstico funcional de los pacientes a su cuidado.

En Chile se producen aproximadamente 25.000 nuevos ACV cada $a n ̃ 0^{2}$, de éstos se estima que entre 5000 y 15000 pacientes necesitarán ayuda de algún familiar para realizar sus AVD. El ACV es una enfermedad asociada a una gran morbilidad que no solo repercute sobre la persona afectada, es una enfermedad que causa impacto en el contexto de la persona secuelada, tanto en el ámbito personal como en el socio ambiental. Para desarrollar la intervención se seleccionaron algunas de las problemáticas que afectan a los cuidadores informales de los pacientes secuelados de un ACV: a) Desconocimiento del origen y consecuencias de la enfermedad, b) Desconocimiento en los cuidados domiciliarios adecuados por parte de los familiares secuelados por el ACV, c) Alteraciones afectivas del cuidador informal principal como: estrés, trastornos del sueño, depresión y ansiedad ${ }^{25}$, d) Distribución de la rutina, tiempo limitado de descanso y ocio, e) Falta de asesoramiento y seguimento luego del alta médica por parte de las instituciones de salud ${ }^{11}$. La intervención tuvo buena acogida por parte de los participantes, con una buena adherencia a todo el proceso educativo. La innovación de este trabajo es incluir conceptos necesarios y atingentes a las necesidades de los participantes, entregando herramientas sencillas de autocuidado como relajación, distribución y organización de la rutina y uso del tiempo libre, el cuál permite fortalecer los recursos y habilidades sociales y ocupacionales del sujeto, además de entregarle conocimientos a las necesidades del cuidado de su familiar enfermo. 
En un estudio de seguimiento realizado por Visser - Meily ${ }^{26}$,a 211 cuidadores de personas secueladas de ACV, que fueron evaluados al segundo mes del alta, tiempo similar al que se utilizo en este estudio, mostro que los cuidadores obtuvieron un promedio en el IEC de 7.1 $\pm 3,4$ puntos a los dos meses, a diferencia de nuestro estudio en que los cuidadores presentaron un puntaje $5.88 \pm 3$ ( $\mathrm{p}=0,15$; IC: $-0.455-2.899)$, donde los puntajes iguales o superiores a 7 significan una gran sensación de carga. Los cuidadores que participaron en la intervención presentaron una escasa sobrecarga al finalizar el seguimiento, información confirmada con los resultados obtenidos en IEC y Escala de Zarit. En cambio en el Goldberg-D presentaron resultados similares $(2,21 \pm 1,8$ en nuestro estudio $)$ en comparación con el estudio de Visser- Meily $(2.6 \pm 2.8)^{26}$, esto puede deberse, tal vez por la nueva condición de vida que se deben enfrentar tanto cuidadores como pacientes.

Por otro lado, si consideramos que los pacientes al ingreso de estudio presentaron un puntaje que los clasifica en un grado de dependencia severa $(\mathrm{BI}=42,7 \pm 24)^{27}$ y que éstos variaron a los 2 meses a un nivel de dependencia moderada ( $\mathrm{IB}=72,7 \pm 19,2)$, necesitando mayor asistencia en actividades de movilidad y en cambio logrando una mayor autonomía en las actividades de cuidado personal, se puede inferir que hubo un efecto positivo sobre los cuidadores, ya que los pacientes requirieron menos supervisión del cuidador, disminuyendo el nivel de exigencia en el cuidado. Los resultados obtenidos en el BI, son similares a los encontrados en un estudio epidemiológico en población china y francesa, donde realizaron la primera medición durante los primeras 2 semanas del ACV, y luego a los 3 meses de evolución, esta última medición fue ligeramente más positiva en la población asiática y europea, aunque siguen clasificados como dependencia moderada, posiblemente a que los pacientes fueron evaluados con 1 mes más de evolución que los pacientes de nuestro estudio ${ }^{28}$. De lo anterior se desprende que el nivel de recuperación del familiar y el aporte de la intervención puede haber afectado en la disminución de la sensación de carga de los cuidadores, pero no así en el estado emocional de éstos que son similares a estudios observacionales ${ }^{26}$. 
La mayor fortaleza de nuestro estudio es que la intervención está basada en la realidad de la población chilena, ajustándose a las necesidades de nuestra población. La mayor debilidad de nuestro trabajo, es el reducido número de pacientes/cuidadores que pudieron ser reclutados, limitando así la generalización de esta propuesta de intervención, por otro lado falto la implementación de un grupo comparador, para determinar la potencia de la intervención. Creemos necesario ahondar en elementos descriptivos y narrativos de los cuidadores, para conocer de cómo es impacto y modificación de su nueva vida.

\section{CONCLUSIÓN.}

Este estudio plantea que nuestra intervención puede ser una herramienta económica y eficiente en la disminución de la sensación de carga y estrés emocional de los cuidadores informales que deben hacerse cargo de sus familiares secuelados de un ACV.

\section{AGRADECIMIENTOS}

Este estudio fue financiado por la Oficina de apoyo a la investigación Clínica (OAIC) del Hospital Clínico Universidad de Chile mediante fondos concursables. 


\section{REFERENCIAS BIBLIOGRÁFICAS}

1. WHO. Burden of Disease Statistics. Geneva, Switzerland: World Health Organization. http://www.who.int/healthinfo/bod/en/index. html (accessed May 2, 2008).

2. LAVADOS, PM. SACKS, C. PRINA, L, et al. Incidence, 30-day case-fatality rate, and prognosis of stroke in Iquique, Chile: a 2-year community based prospective study (PISCIS project). Lancet 2005; 365: 2206-15.

3. KALRA, L. EVANS, E. PEREZ, I. MELBOURN, A. PATEL, A. KNAPP, M. DONALDSON, D. Training carers of stroke patients: randomized controlled trial. BMJ. 2006; 328: 1-5.

4. LAVADOS, P. HOPPE, A. Unidades de tratamiento del ataque cerebrovascular (UTAC) en Chile. Rev. méd. Chile [online]. 2005; 133 (11): 1271-1273

5. DÍAZ-TAPIA, V. GANA, J. SOBARZO, M. JARAMILLO-MUÑOZ, A. ILLANES-DÍEZ, S. Estudio sobre la calidad de vida en pacientes con accidente vascular cerebral isquémico. Rev Neurol. 2008; 46 (11): 652-655

6. LINCOLN, N. FRANCIS, V. LILLEY, S. SHARMA, J. AND SUMMERFIELD, M. Evaluation of a Stroke Family Support Organiser: A Randomized Controlled: Trial. Stroke. 2003;34:16-121

7. LANGHORNE, P. TAYLOR, G. MURRAY, G. DENNIS, M. ANDERSON, C. BAUTZ-HOLTER, E. DEY, P. INDREDAVIK, B. MAYO, N. POWER, M. RODGERS, H. RONNING, OM. RUDD, A. SUWANWELA, N. WIDENHOLMQVIST, L. WOLFE, C. . Early supported discharge services for stroke patients: a meta-analysis of individual patients' data. Lancet. 2005; 365: 501-06

8. BAKAS, T. AUSTIN, JK. OKONKWO, KF. LEWIS, RR. CHADWICK, L. Needs, concerns, strategies, and advice of stroke caregivers the first 6 months after discharge. J Neurosci Nurs. 2002;34(5):242-51

9. JULLAMATE, P. DE AZEREDO, Z. PÁUL, C. SUBGRANON, R. . O Thai stroke patient caregivers: who they are and what they need. Cerebrovasc Dis. 2006;1(12):128-33.

10. PIERCE, LL. GORDON, M. STEINER, V. Families dealing with stroke desire information about self-care needs. Rehabil Nurs. 2004; 29(1):14-7.

11. RODGERS, H. BOND, S. CURLESS, R. Inadequacies in the provision of information to stroke patients and their families. Age and Ageing. 2001; 30:129133

12. TALBOT, LR. VISCOGLIOSI, C. DESROSIERS, J. VINCENT, C. ROUSSEAU, J. ROBICHAUD, L. Identification of rehabilitation needs after a stroke: an exploratory study. Health and Quality of Life Outcomes. 2004; 2(53) ; 1-9

13. SUHA, M. KIMA, K. KIMB, I. CHOC, N. CHOID, N. NOHE, S. Caregiver's burden, depression and support as predictors of post-stroke depression: a crosssectional survey. International journal of Nursing Studies. 2005; 42 : 611-618.

14. SUMMERFIELD, M. LINCOLN, N. FRANCIS, V. LILLEY, S. SHARMA, J .Trial Evaluation of a Stroke Family Support Organiser: A Randomized Controlled. Stroke 2003;34:116-121 
15. BERG, A. PALOMÄKI, H. LÖNNQVIST, J. LEHTIHALMES, M. KASTE, M. Depression Among Caregivers of Stroke Survivors. Stroke 2005;36:39-643

16. RODGERS, H. ATKINSON, C. BOND, S. SUDDES, M. DOBSON, R. CURLESS, R. Randomized Controlled Trial of a Comprehensive Stroke Education Program for Patients and Caregivers. Stroke. 1999;30:2585-2591

17. GRANGER CV, DEVIS LS, PETERS MC, SHERWOOD CC, BARRETT JE. Stroke rehabilitation: analysis of repeated Barthel Index measures. Arch Phys Med Rehabil. 1979;60:14 -17.

18. VAN SWIETEN, JC. KOUDSTAAL, PJ. VISSER, MC. SCHOUTEN, H. VAN GIJN, J. Interobserver agreement for the assessment of handicap in stroke patients. Stroke. 1988;19:604-607

19. BONITA, R. BEAGLEHOLE, R. Recovery of motor function after stroke. Stroke. 1988;19:1497-1500.

20. RANKIN, J. Cerebral vascular accidents in patients over the age of 60. Scott Med J. 1957;2:200-215

21. MONTORIO, I. IZAL FERNÁNDEZ DE TROCÓNIZ, M. LÓPEZ, A. SÁNCHEZ, M. La Entrevista de Carga del Cuidador. Utilidad y validez del concepto de carga. Anales de psicología. 1998; 14(2): 229-248.

22. ROBINSON, BC. Validation of a caregiver strain index. J Gerontol. 1983; 38: 344348.

23. VISSER-MEILY, JM. POST, MW. RIPHAGEN, II. LINDEMAN, E. Measures used to assess burden among caregivers of stroke patients: a review. Clin Rehabil. 2004; 18: 601-623.

24. GOLDBERG, D. HOFFMAN, A. FUROMOTO-DAWSON, A. NELSONJOHNSON, H. Mindfulness-based stress reduction and its effects on well-being. J Investig Med. 1998;46:278 A.

25. VISSER-MEILY, A. VAN HEUGTEN, C. POST, M. SCHEPERS, V. LINDEMAN, E. Intervention studies for caregivers of stroke survivors: a critical review. Patient Education and Counseling. 2005; 56: 257-267.

26. VISSER-MEILY, A. VAN HEUGTEN, C. POST, M. SCHEPERS, V. LINDEMAN, E Psychosocial functioning of spouses of patients with stroke from initial inpatient rehabilitation to 3 years poststroke: course and relations with coping strategies. Stroke. 2009;40(4):1399-404.

27. SHAH, S. VANCLAY, F. COOPER B. Improving the sensitivity of the Barthel Index for stroke rehabilitation. J Clin Epidemiol 1989: 42: 703-709.

28. ZHOU, Z. DAVIET, JC. MARIN, B. MACIAN, F. SALLE, JY. ZHOU, N. ZHU, Y. Vital and functional outcomes of the first-ever hemispheric stroke, epidemiological comparative study between Kunming (China) and Limoges (France). Ann Phys Rehabil Med. 2010; 53(9):547-58. 